\title{
Morphology of Perylene Dimide based Polymer Non-Fullerene Solar Cells: Effect of Thermal Annealing
}

Tia Wright ${ }^{1}$, Washat Ware ${ }^{1}$, Jennifer Barkley ${ }^{1}$, Shubo Han $^{2}$ and Bhoj Gautam ${ }^{1}$

${ }^{1}$ Fayetteville State University, Fayetteville, North Carolina, United States, ${ }^{2}$ Fayetteville State University, FAYETTEVILLE, North Carolina, United States

Conjugated polymers have emerged as an interesting class of photovoltaic materials due to their unique properties including light weight, mechanical flexibility and compatibility with roll-to-roll, and non-toxic processing methods. Fullerene derivatives have been extensively used as acceptor materials of choice in organic solar cells (OSC) since the early 1990s but with several drawbacks such as higher production cost, limited absorptivity and spectrum coverage [1], open circuit voltage loss[2], etc. Recently, considerable efforts have been dedicated to the development of non-fullerene acceptors[3]. These acceptors have several advantages over fullerene derivatives, namely high absorption coefficient [4], near infra-red absorption, highly tunable molecular energy levels [4-8], low voltage loss, and complementary donor acceptor absorption[911]. To the date, power conversion efficiency of $16.5 \%$ [12] has been reported using these acceptors. There are several factors that influence charge generation and transport in bulk heterojunction OSCs including morphology. In this work, we used perylene diimide (PDI)[13]-based non fullerene acceptor called SF-PDI (PDI dimer with spirofluorene linker) and donor polymer PBDTBDD-T (poly[(2,6-(4,8-bis(5-(2ethylhexyl)thiophen-2-yl)-benzo[1,2-b:4,5-b']dithiophene))-alt-(5,5-(1',3'-di-2-thienyl-5',7'-bis(2ethylhexyl)benzo[1',2'-c:4',5'-c']dithiophene-4,8-dione))]) abbreviated as PBDB-T to prepare the bulk heterojunction. Using Atomic Force Microscopy (AFM) in tapping mode, we studied the impact of thermal annealing on the morphology and phase separation in PBDB-T:SF-PDI blend. The donor polymer PBDB-T and non -fullerene acceptor SF-PDI were purchased from 1-Materials and used as received without further purification. Blend solution of PBDB-T:SF-PDI $(1: 1 \mathrm{w} / \mathrm{w})$ was prepared by dissolving in chlorobenzene and stirring at $800 \mathrm{C}$ for 12 hours. The concentration of blend solution was $10 \mathrm{mg} / \mathrm{ml}$. The glass substrates were cleaned ultrasonically using deionized water, acetone, and isopropanol for $15 \mathrm{~min}$ per cleaning solvent before spin casting. Blend films were prepared by spin casting the solution on glass substrates at $1000 \mathrm{rpm}$ for $60 \mathrm{~s}$. Three thin films were prepared and two of them were annealed at $900 \mathrm{C}$ and $1300 \mathrm{C}$. The AFM images shown in Figure 1 and 2 indicate that there is a contrast in the morphology of annealed and non-annealed blends. Figure $1(\mathrm{a}, \mathrm{b})$ shows the atomic force microscopy topography image of PBDB-T:SF-PDI blends at different annealing conditions. The topography images of non-annealed PBDB-T:SF-PDI film reveal the rough surface with root mean square roughness $(\mathrm{Rq})$ in the range of $6.37 \mathrm{~nm}$. Roughness increases upon annealing to 17.7 $\mathrm{nm}$ at $900 \mathrm{C}$ and $27.2 \mathrm{~nm}$ at $1300 \mathrm{C}$. AFM phase images (Figure $2 \mathrm{a}, \mathrm{b}, \mathrm{c}$ ) indicate that more distinct phase separated domains are observed in annealed blended thin films compared to non-annealed one. Our results indicate that the annealing improves the nanomorphology of PBDB-T:SF-PDI blend. This difference in morphology and phase separation can result the difference in performance of these solar cells. Corresponding Author: *bgautam@uncfsu.edu Acknowledgments: This work was supported by NSF RIA (HRD 1900998) and EIR (ECCS-1900837). 


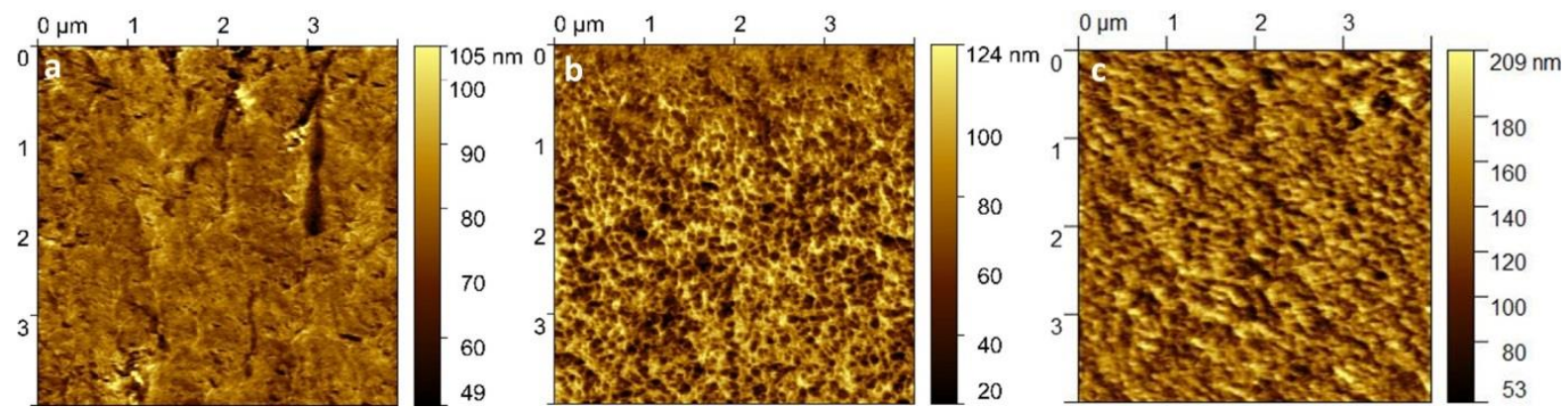

Figure 1. Figure 1. Atomic force microscopy topography image of (a) non-annealed PBDB-T:SF-PDI film (b) PBDB-T:SF-PDI film annealed at $900 \mathrm{C}$ and PBDB-T:SF-PDI film annealed at $1300 \mathrm{C}$.

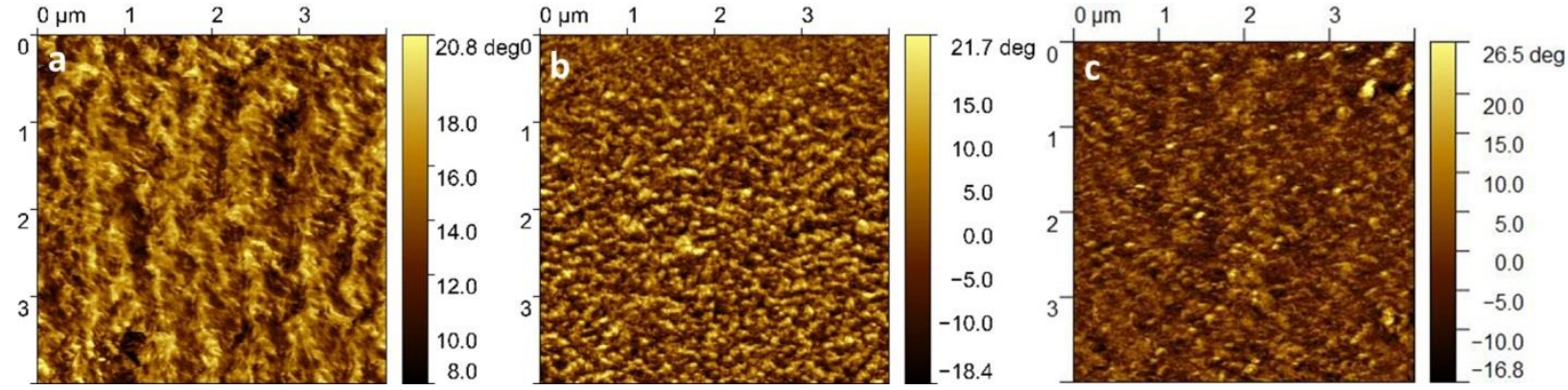

Figure 2. Figure 2. Atomic force microscopy phase image of (a) non-annealed PBDB-T:SF-PDI film (b) PBDB-T:SF-PDI film annealed at $900 \mathrm{C}$ and PBDB-T:SF-PDI film annealed at $1300 \mathrm{C}$.

\section{References}

[1] V. Gupta, et. al., Adv. Mater., 27 (2015) 4398-4404.

[2] J. Wang, et. al., J. Mater. Chem. A, 6 (2018) 19934-19940.

[3] Z. Zhang, et. al., Front. Chem., 6 (2018) 414.

[4] C.B. Nielsen, et. al., Accounts of chemical research, 48 (2015) 2803-2812.

[5] Y. Lin, et. al., J. Am. Chem. Soc, 138 (2016) 2973-2976.

[6] Y. Lin, et. al., Energy \& Environmental Science, 8 (2015) 610-616.

[7] Y. Lin, et. al., J. Am. Chem. Soc, 138 (2016) 4955-4961.

[8] Z. Zheng, et. al., Adv. Mater, 29 (2017).

[9] J. Liu, et. al., Nat. Energy, 1 (2016) 16089.

[10] O.K. Kwon, et. al., Adv. Mater, 28 (2016) 910-916.

[11] N. Gasparini, et. al., Adv. Energy Mater. 8 (2018).

[12] Y. Cui, et. al., Nat. Commun., 10 (2019) 2515.

[13] J. Zhang, et. al., J. Am. Chem. Soc, 139 (2017) 16092-16095. 